\title{
ILCEA
}

Revue de l'Institut des langues et cultures

d'Europe, Amérique, Afrique, Asie et Australie

$30 \mid 2018$

Création culturelle et territoires : de l'histoire au

mythe, du réel à l'utopie

\section{Utopie révolutionnaire et utopie conservatrice : la réception politique des textes utopiques italiens de la Renaissance}

Revolutionary and Conservative Utopia: The Political Reception of Italian

Renaissance Utopian Literature

\section{Federica Greco}

\section{OpenEdition}

Journals

Édition électronique

URL : http://journals.openedition.org/ilcea/4599

DOI : $10.4000 /$ ilcea.4599

ISSN : 2101-0609

Éditeur

UGA Éditions/Université Grenoble Alpes

Édition imprimée

ISBN : 978-2-37747-033-4

ISSN : $1639-6073$

Référence électronique

Federica Greco, «Utopie révolutionnaire et utopie conservatrice : la réception politique des textes utopiques italiens de la Renaissance », ILCEA [En ligne], 30 | 2018, mis en ligne le 31 janvier 2018, consulté le 10 décembre 2020. URL : http://journals.openedition.org/ilcea/4599; DOI : https://doi.org/ 10.4000/ilcea.4599

Ce document a été généré automatiquement le 10 décembre 2020.

(c) ILCEA 


\title{
Utopie révolutionnaire et utopie conservatrice : la réception politique des textes utopiques italiens de la Renaissance
}

\author{
Revolutionary and Conservative Utopia: The Political Reception of Italian \\ Renaissance Utopian Literature
}

Federica Greco

1 Nous appartenons tous à l'espèce de l'homo utopicus. Dès que l'homme a ressenti le besoin de changer sa situation politique et sociale, il a commencé à concevoir des modèles communautaires alternatifs (Quarta, 2015). L'utopie serait donc une catégorie anthropologique, un phénomène humain : nous avons tous une conscience utopique, pour utiliser la terminologie de Melchiorre (1970), qui nous pousse à imaginer des changements afin d'améliorer notre réalité et le monde dans lequel nous vivons. À partir des premières grandes études allemandes sur l'utopie - Der Geist der Utopie (1918) d'Ernst Bloch et Ideologie und Utopie (1929) de Karl Mannheim - la critique s'est interrogée sur le rapport entre utopie et réalité historique ${ }^{1}$. Il semblerait que ce soit l'utopie même, avec la possibilité qu'elle nous donne de transcender la réalité, qui donne l'impulsion à tout bouleversement ayant déterminé notre Histoire. En ce sens, les projets politiques qui visent à la préservation d'un statu quo, à la conservation d'un équilibre, et qui refusent donc les oppositions qui sont à la base de la progression de l'histoire, ne peuvent pas être considérés comme des utopies.

2 Si nous acceptons cette définition, nous pouvons nous demander quelle est donc, dans ces théories, la place des œuvres littéraires de la Renaissance qui ont fondé le genre utopique et lui ont donné son nom ambigu, qui nous force à nous interroger en permanence sur le rapport entre ce qui existe et ce qui n'existe pas ${ }^{2}$. Le caractère irrémédiablement figé de l'œuvre littéraire, ainsi que les renvois intertextuels à l'antiquité classique, notamment à la République de Platon et aux mythes de l'âge d'or, ont conféré à ces projets une 
dimension moins révolutionnaire que conservatrice, ce qui les exclurait automatiquement de la définition d'«utopie». Pour ne pas tomber dans le paradoxe d'écarter de ce domaine de recherche les textes mêmes qui ont contribué à forger cette catégorie de la pensée humaine, certains critiques $^{3}$ ont défini ces ouvrages comme de premiers essais imparfaits, un pâle reflet d'une tension anthropologique, philosophique et historique beaucoup plus complexe. Toutefois, si nous considérons l'historiographie des $\mathrm{XIX}^{\mathrm{e}}$ et $\mathrm{XX}^{\mathrm{e}}$ siècles, nous nous apercevons que les œuvres utopiques de la Renaissance ont été souvent centrales dans les enquêtes sur l'histoire de la pensée politique, au point d'avoir créé une spécificité de la pensée utopique italienne, parallèle à celle de grands penseurs comme Machiavel ou Guichardin.

Nous nous arrêterons en particulier sur une polémique entre Benedetto Croce et certains représentants du matérialisme historique à propos de l'interprétation de ces ouvrages ainsi que sur la création progressive d'un corpus de textes utopiques dans les anthologies des écrivains politiques italiens $\mathrm{du} \mathrm{XVI}^{\mathrm{e}}$ et du XvII ${ }^{\mathrm{e}}$ siècle. Enfin, nous nous demanderons si cette tension entre une vision exclusivement littéraire des œuvres utopiques, qui les relègue dans le monde de la fiction et de l'imaginaire, et une vision politisée, ne serait pas un réflexe d'un dualisme déjà présent dans la forme de l'utopie telle qu'elle a été conçue par Thomas More.

\section{Interprétation socialiste et matérialisme historique}

4 Les œuvres utopiques de la Renaissance italienne ont été souvent employées par les socialistes pour prouver que le désir d'améliorer la société est inhérent à l'esprit humain, comme l'explique François Villegardelle à propos de la Cité du Soleil de Campanella :

Elle offre, avec la doctrine saint-simonienne ainsi qu'avec la théorie de Fourier, des analogies tellement frappantes, qu'un examen superficiel ferait soupçonner un plagiat. Mais une étude approfondie des trois systèmes rend, à nos yeux, cette supposition tout-à-fait inadmissible. Ce n'est donc pas pour nous donner le triste plaisir de mettre en suspicion la bonne foi des réformistes contemporains, que nous publions la traduction de La Cité du Soleil. Notre unique but a été de continuer l'œuvre que nous avons commencée en publiant Le Code de la Nature, de Morelly; c'est-à-dire de prouver que l'esprit humain, dont la nature est toujours la même, n'a cessé d'opposer aux souffrances d'une société vicieuse le type idéal d'une société plus parfaite; de montrer ensuite aux différentes écoles socialistes les racines qu'elles ont dans le passé, et de les rappeler ainsi à des sentiments moins dédaigneux et moins exclusifs. (Villegardelle, $1840: 14$ )

5 L'utopie et le socialisme partageraient donc une même tension vers le changement, un besoin d'opposition aux injustices sociales, qui sont devenues plus tard l'objet des analyses philosophiques et anthropologiques sur l'utopisme. Cet avis est partagé par des théoriciens du socialisme, comme Benoit Malon, qui affirme que l'idéal d'une société égalitaire est une partie intégrante de la nature humaine, comme le démontre la persistance de certaines thématiques à travers les siècles. Dans ses précis historiques - en particulier Le Socialisme intégral ; Le Précis historique, théorique et pratique de socialisme; Histoire du socialisme depuis les temps les plus reculés jusqu'à nos jours - il accorde une grande importance aux premiers textes utopiques modernes, perçus comme les précurseurs du socialisme. À côté des œuvres principales du genre utopique, notamment l'Utopie de More et La Cité du Soleil de Campanella, y figurent aussi des ouvrages italiens mineurs, comme Les Mondes (1552) d'Anton Francesco Doni ou La République des Abeilles (1627) de Giovanni 
Bonifacio. Des Mondes a été extrait le dialogue entre le Sage et le Fou qui a eu une grande fortune autonome dans l'histoire de l'utopie. Doni y décrit le rêve d'un «monde nouveau » où l'on ressent beaucoup l'influence de l'Utopie de More et sa description de la cité idéale est accompagnée également par des réflexions sur les injustices sociales de son époque. Dans La République des Abeilles, nous retrouvons le topos de la découverte d'une société alternative lors des explorations géographiques: des navigateurs portugais, envoyés en Amérique sous le règne de Jean III, signalent à leur retour l'existence d'une population vivant encore à l'état de nature mais, au lieu de prendre exemple sur elle comme il advient dans la plupart des utopies, le roi Philippe II décide de les forcer à se convertir au christianisme et à la civilisation. Interrogés sur le bon gouvernement qu'on devrait leur imposer, les explorateurs proposent une «république populaire » sur le modèle de la vie des abeilles présentée par Virgile dans le quatrième livre des Géorgiques. La République des Abeilles semble donc n'être proche du genre de l'utopie que par son seul récit-cadre, mais il est évident que Malon n'a pas choisi ses textes en raison d'une cohérence littéraire ou philosophique mais pour les contenus qui renvoient à l'idéal d'une société communautaire et équitable et, en définitive, au socialisme.

Interpréter ces œuvres à la lumière de la pensée politique de la fin du XIX ${ }^{e}$ siècle nous semble aujourd'hui anachronique et tendancieux mais, pour Malon, étudier la façon dont les idées de justice sociale se sont succédé au fil des siècles n'était pas anodin dans une conception du monde comme "branloire pérenne ${ }^{4}$ ». Cela permettait aussi de mieux comprendre la transmission des idées et la succession d'événements ayant conduit à la situation politique de son temps (Malon, 1892b). Reprenant les mots d'Émile de Lavelay (1883:v), il affirmait que les aspirations socialistes, bien que filles de la Révolution française, « tantôt sous forme de protestation contre le mal existant, tantôt sous celle de plans utopiques de reconstruction sociale, se firent jour dès que l'homme eut assez de culture pour ressentir les iniquités sociales » (Malon, 1892a : 86). Il n'est pas loin de l'idée d'une utopie anthropologique comme l'a définie Quarta, mais sa perspective centrée sur le socialisme et son histoire finit par lui faire voir le socialisme et l'utopie comme deux catégories interchangeables, au lieu de concevoir le mouvement politique comme l'une des réalisations possibles de l'utopie (Quarta, 2016).

7 L'approche politisée de Malon ne fut pas un cas isolé : Emilio Bertana (1892) intitula Un socialista del Cinquecento son essai sur Anton Francesco Doni. Bien qu'il mette en garde son lecteur contre le risque d'anachronismes - et surtout contre l'idée que Doni pourrait être un précurseur des socialistes contemporains -, il dit avoir repéré dans ses œuvres, notamment dans Les Mondes, des passages ayant beaucoup de points communs avec les principes du socialisme. Les critiques adressées par Doni contre les injustices sociales semblent coller parfaitement à un esprit de la fin du XIX ${ }^{e}$ siècle. Il aurait ainsi, par exemple, dénoncé l'inéquitable répartition des richesses, bien avant Brissot ou Proudhon:

[...] c'est un homme nouveau, il utilise un langage qui ne semble pas appartenir à son époque, et il produit en nous, avec ces étranges accents de colère et de pitié, l'illusion d'entendre une des voix les plus caractéristiques de notre siècle ${ }^{5}$. (1892: 359)

En conclusion de ces interprétations socialistes, nous pouvons aussi citer l'essai d'Andrea Calenda di Tavani (1895), homme politique et sénateur italien, qui s'inscrit dans la même lignée : Tommaso Campanella e la sua dottrina sociale e politica di fronte al socialismo moderno. 
9 Mais au-delà des points communs, il y avait sûrement des aspects de ces cités imaginaires qui ne pouvaient être compatibles avec les principes du socialisme et Malon critique, par exemple, l'esclavage et la peine capitale dans l'Utopie ou la vision des femmes de la part de Campanella. Toutefois, les socialistes n'ont pas tous fait le rapprochement avec les textes utopiques en raison seulement d'une proximité dans l'histoire des idées : Lafargue (1895) chercha en effet à appliquer les notions du matérialisme historique à la Cité du Soleil. Bien que «la fin de l'utopie » et le passage au socialisme scientifique avaient été proclamés, l'intérêt pour les œuvres utopiques ne s'estompa pas pour autant. Selon la théorie historiographique du matérialisme historique formulée par Marx et Engels, tous les faits historiques sont explicables en dernière instance par la structure économique sousjacente. Il faut toutefois opérer une médiation entre les faits économiques, dont les protagonistes des changements historiques ne sont pas forcément conscients, et la conscience spécifique - ce que nous pourrions appeler communément la pensée - des hommes dans un certain contexte social (Labriola, 1970). Or Lafargue a, au contraire, appliqué le principe du matérialisme historique comme une formule rigide, en affirmant que Campanella, dans son projet de la cité idéale,

[...] exprimait, sans le savoir, d'une manière philosophique, l'impérieux besoin économique de la Bourgeoisie capitaliste de son temps. En effet, elle ne pouvait se développer économiquement et politiquement qu'à la condition de détruire l'autonomie des villes et des provinces pour élever sur leurs ruines les unités nationales, qui n'ont achevé leur construction que de nos jours ; - de renverser les barrières locales et provinciales qui entravaient et empêchaient même la libre circulation des marchandises; - d'abolir les privilèges locaux et corporatifs qui s'opposaient à l'établissement de l'industrie manufacturière ; - d'imposer aux rois et aux seigneurs féodaux qui battaient monnaie et la falsifiaient, le respect de la valeur de l'argent et de l'or ; - et d'établir une unification des poids et des mesures, dont la variabilité gênait les échanges d'une localité à une autre. (Lafargue, 1895 : 477)

Si l'analyse de la naissance de la bourgeoisie opposée au système féodal et marquant les prodromes du capitalisme peut être partagée, les idées de Campanella passent définitivement à l'arrière-plan comme l'expression naïve d'une société qui n'avait pas encore les moyens pour s'exprimer de façon efficace. Le même sort a été réservé aux hérésies et aux réformes théologiques, réputées n'être que les signes d'un besoin économique, et de la volonté de déposséder l'Église de ses bien matériels. Plus loin, Lafargue en vient même à faire une comparaison entre la théorie marxiste de la plusvalue et les théories de la Kabbale que, selon une des nombreuses légendes, Campanella avait appris d'un vieux rabbin alors qu'il séjournait dans le couvent des dominicains de Cosenza :

Le panthéisme et la transmigration des âmes de la Kabbale ne sont que des expressions métaphysiques de la valeur des marchandises et de leur échange. La valeur, ainsi que l'Être qui vit dans toute chose créee, est incorporée dans tout ce qui se vend et s'achète; toute marchandise possède une quantité déterminée de valeur, comme toute chose animée ou inanimée participe à des degrés divers aux attributs de l'Être. La valeur d'une marchandise transmigre dans une autre, puisque dans une marchandise revit la valeur de la matière première et des instruments qui ont concouru à sa production. Toutes les marchandises bien que différentes de qualité expriment cependant leur valeur, différente en quantité, dans l'argent, qui devient la marchandise par excellence, celle qui personnifie l'unité des marchandises. Marx a démontré que l'échange capitaliste débute par l'argent pour aboutir à l'argent, mais à l'argent avec un incrément : la théosophie de la Kabbale part de l'unité, le $1^{\text {er }}$ Séphiroth, pour aboutir avec le $10^{\mathrm{e}}$ Séphiroth à l'unité 
complexe, puisqu'il accumule les attributs des 9 Séphiroths précédents. (Lafargue, 1895 : 477-488)

11 On touche avec cette citation le sommet des dérives interprétatives qui ont vidé ces textes utopiques de toute valeur spécifique par rapport à leur contexte historique, social et politique. Mais si ces théories nous paraissent aujourd'hui des bizarreries, elles eurent pourtant un rôle dans l'histoire des textes utopiques ne serait-ce qu'en raison des fortes réactions qu'elles entraînèrent chez Benedetto Croce.

12 Ce dernier s'exprima à propos de cette tendance historiographique dans un article intitulé "Sulla concezione materialistica della storia ${ }^{6}$ " où il dénonçait une distorsion opérée par certains historiens dans l'application du matérialisme historique tel qu'il avait été formulé par Marx et Engels, et où il répondait directement à Lafargue à propos de la question de "Campanella communiste ${ }^{7}$ ». Nous ne nous arrêterons pas sur les critiques concernant les connaissances historiques et littéraires de Lafargue, qui avait fait des études de médecine, car ses limites dans ses domaines ne sont que trop évidentes; ce qui nous intéresse, outre la condamnation de cette méthodologie, est surtout l'exclusion nette de ces textes de l'histoire de la pensée politique. Selon Croce, ces quelques expériences révolutionnaires, qui n'ont eu aucune conséquence, ou presque, dans la réalité historique, ont été liées entre elles arbitrairement en prenant en compte des auteurs dont la pensée n'a rien à voir avec les événements historiques. S'il accorde une certaine importance à l'Utopie, car son auteur avait produit une analyse lucide de la situation sociale de l'Angleterre de son temps, il pense, au contraire, que le communisme de Campanella s'inspirait d'une observation générale de l'injustice sociale comme on peut en trouver à toutes les époques. Son utopie était donc moins alimentée par une conscience de la situation économique et sociale dans laquelle il se trouvait que par une mission providentielle dont il se sentait le dépositaire. Au-delà de l'amorce d'un programme de gouvernement théocratique, destiné à se répandre à l'échelle universelle, Croce ne repère aucune solidité concrète dans la pensée politique de Campanella, étant donné que même sa tentative révolutionnaire en Calabre resta sur le seul plan théorique. Les approches des critiques socialistes, qui voyaient dans les utopies de la Renaissance une étape capitale dans la formation de la pensée politique et dans l'histoire du communisme, ont été donc complètement renversées par Croce qui considérait ses projets comme purement abstraits :

L'œuvre du politique consiste non pas dans des constructions plus ou moins ingénieuses et remarquables au point de vue esthétique ou moral, mais à trouver les points de contact de l'idéal et de la réalité. Et la raison qui fait que les utopies n'ont qu'un faible intérêt pour la science politique, c'est précisément que les moyens sont complètement négligés ou purement fantaisistes. Or, de tous les utopistes, qui fut plus utopiste que Campanella? (Croce, 1901 : 303)

Le seul mérite des utopies fantaisistes comme celle de Campanella aurait été d'avoir montré aux socialistes qu'ils ne devaient pas tomber dans la même erreur pour pouvoir réaliser le passage du socialisme "utopique " au socialisme "scientifique ". Toutefois, quelques années plus tard, Croce est revenu sur la question en considérant le communisme en lui-même comme une utopie à cause de son idéal d'une société sans classes où toute opposition et oppression auraient été effacées. La revendication d'un dépassement du socialisme utopique ne serait alors qu'illusion. C'est dans ce contexte que nous devons lire la citation de Croce qui éloigne l'utopie de l'histoire :

En quoi consiste réellement l'utopie? On dit que l'«utopie d'aujourd'hui sera

l'histoire de demain "; et dans ce sens l'utopie n'est rien d'autre que le dessin de 
quelque chose de possible qui aujourd'hui ne trouve pas les moyens nécessaires pour se réaliser et les trouvera ou pourra les trouver plus tard. Mais ce n'est pas la véritable utopie qui est ce qui n'est " en aucun lieu », soit comme on dit « qui est en dehors de l'histoire ", de toutes les histoires, des conditions de toutes les histoires, et qui nie l'histoire puisqu'elle a tendance à la stase, tandis que l'histoire est mouvement, et le mouvement est la dialectique des oppositions. Toutes les tentatives pour chasser les oppositions de l'histoire est inutile ; toute conception où les oppositions sont éliminées est contradictoire et vide : c'est-à-dire utopique ${ }^{8}$. (Croce, $1948: 24$ )

En reprenant la communis opinio que "l'utopie d'aujourd'hui sera l'histoire de demain ", dont les socialistes ont fait grand usage en attribuant cette citation à différents hommes illustres, il affirme qu'il ne pourrait s'agir de la véritable utopie qui, par définition, ne pourra jamais se réaliser. L'utopie est conservatrice, car elle conçoit une société sans inégalités ni oppositions où tout aurait pour finalité le maintien du statu quo, ce qui serait contraire à l'évolution naturelle de l'histoire. Cette théorie semble en opposition avec l'idée d'une utopie comme moteur des révolutions et des changements historiques, ou avec l'idée de Mannheim présentant l'utopie comme rupture effectuée par les classes subalternes par rapport à la réalité présente. En réalité, la différence principale est déterminée par une perspective uniquement terminologique: Croce définit l'utopie en prenant comme point de départ les œuvres de Campanella et des autres utopistes de la Renaissance pour définir toutes les idées qui ne sont pas "agissantes» (Croce, 1968: 221-226); d'autres théories débutent en revanche leur analyse de l'utopie dans la conscience et dans le comportement humain dont les œuvres littéraires ne sont qu'un reflet ne pouvant entièrement traduire cette véritable tension. Ces deux perspectives différentes parviennent finalement à la même conclusion: les œuvres littéraires utopiques n'ont pas leur place dans les évolutions déterminantes de notre histoire.

\section{La construction d'un corpus d'œuvres italiennes utopiques et politiques}

Malgré cette coupure nette entre l'utopie et l'histoire dans les théories critiques à cheval entre les $\mathrm{XIX}^{\mathrm{e}}$ et $\mathrm{XX}^{\mathrm{e}}$ siècles, on remarque que dans les études littéraires les utopies continuèrent à être présentées comme des œuvres politiques à côté de Machiavel, Guichardin ou Sarpi. Sous le fascisme, la volonté de valoriser l'histoire et la culture italiennes a entraîné la naissance, en 1941, d'une collection intitulée " Écrivains politiques italiens ", créée par l'Institut national de culture fasciste (INCF), sous la direction de son directeur adjoint Guido Mancini puis de Felice Battaglia (professeur de philosophie à l'université de Bologne). À côté d'auteurs politiques comme Alamanni, Della Casa ou Alberti ${ }^{9}$, a été publié un recueil d' «utopistes et réformateurs sociaux $\mathrm{du} \mathrm{xVI}^{\mathrm{e}}$ siècle " (Curcio, 1941). Comme on peut le lire sur le rabat de la couverture, cette collection « vise à divulguer la tradition glorieuse de la pensée politique nationale à travers les textes de nos meilleurs écrivains, présentés dans des éditions corrigées et parfois critiques et accompagnées par des préfaces et des notes explicatives sous la direction d'écrivains contemporains éminents ${ }^{10} »$. Il est assez étonnant de voir parmi ces écrivains les œuvres utopiques d'auteurs mineurs comme Doni et son dialogue entre le Sage et le Fou, déjà brièvement mentionné, Uberto Foglietta (Delle cose della Repubblica di Genova, 1559), Francesco Patrizi (La città felice, 1553) et Ludovico Agostini (La Repubblica immaginaria, extraite de L'Infinito, 1580 ?). Le dialogue de Foglietta est une reconstruction nostalgique 
de l'ancienne gloire de la république de Gênes qui, avant que les disputes internes ne la fassent tomber, était administrée par les citoyens sans distinction de classe, ce que l'auteur prouve avec des documents d'archives. L'œuvre provocatrice de l'historien génois, qui lui coûta l'exil, s'insère dans le débat sur la situation politique de sa ville, mais elle n'avance aucune solution pratique d'amélioration, sinon l'idée générale d'une république anti-nobiliaire. Même dans la « cité heureuse » de Patrizi nous ne retrouvons pas la forme fictionnelle typique du roman utopique, mais l'auteur fait une description minutieuse de la société idéale dont les derniers livres de La Politique d'Aristote sont la source principale. Enfin, Agostini imagine la ville parfaite selon les idéaux religieux et moraux de la Contre-Réforme, une position opposée à celle de la plupart des utopies de la Renaissance qui aboutissaient à une religion libre et naturelle (Firpo, 1957). Selon l'éditeur du volume, ces textes si différents sont liés entre eux par la recherche d'une vie politique heureuse qui puisse réconforter davantage que la vision réaliste de Machiavel et Guicciardini. Si d'une part ils semblent être de simples exercices littéraires, de l'autre Curcio y voit aussi une exigence de renouvellement et un élan vers l'action. Giuseppe Bottai, ministre de l'Éducation sous le fascisme entre 1936 et 1943, insérait l'Utopie de More parmi les chefs-d'œuvre littéraires qui ont créé l'homme non comme être mais comme homo faber. Dans cette perspective, c'est donc grâce à ses actions, que l'homme parvient aussi à changer sa façon d'être (Bottai et De Luca, 1989: 61). Après l'exclusion prononcée par Croce, les textes utopiques reviennent donc faire partie de ces forces motrices qui ont forgé l'Histoire.

Bien évidemment, nous ne pouvons pas retracer ici l'histoire de toutes les publications des textes de la Renaissance considérés comme utopiques, ni de toutes les études critiques de quelque importance qui ont suivi, mais il est intéressant de noter qu'en 1964 Bruno Widmar reprend quasiment le même corpus d'ouvrages établi dans les années 1940 dans son recueil intitulé Scrittori politici del ' $500 e^{\prime} 600^{11}$, avec cependant des ajouts significatifs comme la Cité du Soleil. Bien que le mot «utopie» n'apparaisse pas dans le titre du recueil, qui rassemble «les textes les plus importants de la pensée politique italienne ", l'éditeur dit avoir sélectionné d'une part des œuvres utopiques, de l'autre des textes faisant partie du genre de l'« ottimo Stato ». Selon Widmar, l'utopie, à la différence des théories cherchant des solutions concrètes pour trouver le "bon gouvernement", serait le reflet d'une exigence psychologique de la bourgeoisie italienne qui, face à la suprématie des autres puissances européennes, s'était renfermée dans un idéal de paix. De plus, cette société utopique n'était pas imaginée à partir de la recherche des solutions liées à la réalité historique et n'avait pas une fonction révolutionnaire mais plutôt conservatrice, voire réactionnaire. Les Italiens, enfermés dans leur recherche de la conservation de l'État, n'auraient ainsi été touchés ni par la Réforme, ni par la naissance du capitalisme (Widmar, 1964: 10-14). Cette théorie, contestable sur plusieurs points, compte donc les textes utopiques parmi les ouvrages politiques, sans toutefois leur accorder aucune importance historique ni aucune capacité d'analyse de la situation sociale, de sorte que nous pouvons nous demander pour quelle raison ils ont été insérés dans un recueil des « écrivains politiques les plus importants ».

17 Aujourd'hui, la question ne semble pas complètement résolue, mais il s'est imposé une approche moins inclusive qui, sans renoncer à retracer le cheminement d'une tendance utopique à la Renaissance, étudie chaque ouvrage dans son contexte spécifique. Dans les contributions critiques les plus récentes (Pissavino, 2002) l'utopie et la raison d'État ne sont pas distinguées selon qu'elles coïncident plus ou moins avec la réalité ; au contraire, elles 
ont un but commun : la conservation de l'ordre politique. L'utopie ne serait pas un simple réflexe psychologique amenant l'homme à s'isoler de la réalité connue, mais elle concevrait un processus de rationalisation urbanistique alternative, qui ne s'adresse pas au Prince, comme chez Machiavel et les théoriciens de la raison d'État, mais qui tienne plutôt compte de l'ordonnancement de l'ensemble des citoyens. La recherche d'une société heureuse ne peut pas se passer des valeurs communautaires et du bien-être collectif. Le caractère fictionnel, typique des utopies, permettrait, selon Pissavino, de s'abstraire d'un contexte générique pour en tirer des normes universelles applicables en tout lieu et en tout temps. C'est peut-être justement la forme des premières utopies qui a engendré, comme nous le verrons en conclusion, des interprétations discordantes sur la valeur effective de l'utopie dans le débat politique.

\section{Une forme ambiguë}

18 Déjà Francesco Sansovino avait incorporé l'Utopie de More parmi les formes de gouvernement réelles dans son traité Del governo de' regni e delle repubbliche così antiche come moderne (1561) tout comme le fit Gabriel Chapuys dans L'Estat, Description et Gouvernement des Royaumes et Republiques de Monde, tant anciennes que modernes (1585), un plagiat partiel de l'œuvre de Sansovino (Rivoletti, 2003: 55-57). Tous les deux ne reprennent toutefois que la seconde partie de l'ouvrage de More et cela ne fut pas un cas isolé, comme le remarque Rivoletti. Le premier traducteur de l'Utopie, Claudius Cantiuncula, n'avait pris en considération que cette seconde partie en la proposant comme solution concrète aux problèmes de la ville de Bâle. L'Utopie de More est, en effet, divisée en deux livres : dans le premier il est plutôt question des problèmes sociaux de l'Angleterre de l'époque, alors que dans le second le lecteur est plongé dans le récit fictionnel du navigateur Raphaël Hythlodée et dans sa découverte de l'île d'Utopie pendant son voyage à la suite d'Amerigo Vespucci. La critique s'est longtemps interrogée sur l'équilibre entre l'analyse politique et la partie fictionnelle de l'œuvre et sur le degré de sérieux de More dans la présentation de ce modèle. Aujourd'hui la dimension ludique de l'œuvre a été presque universellement acceptée et il serait naïf de penser que l'auteur considérait l'île d'Utopie comme un modèle directement applicable. Le récit-cadre pouvait avoir pour fonction de rendre universelle la réflexion sur une société égalitaire et heureuse, comme le suggère Pissavino, mais aussi de présenter cette réalité depuis un autre point de vue, qui n'est pas celui de l'essayiste.

Le récit est confié au navigateur portugais Hythlodée, qui en grec signifie « charlatan », et nous invite à considérer la réalité sous une autre perspective, celle de la folie. Cette thématique a été reprise par Doni dans la novella de « la pluie de la folie ${ }^{12}$ » qui précède le dialogue entre le Fou et le Sage et elle a derrière elle une longue tradition littéraire (Rivoletti, 2003 : 19-24). Il faut aussi noter que c'est le Sage, ici, qui rêve d'une ville utopique dans une optique d'interchangeabilité entre ce qui peut être considéré comme une vérité et ce qui peut l'être comme une folie. Même Ortensio Lando, qui en 1548 traduit l'Utopie en italien, est sensible à ce paradigme et dans un ouvrage original publié la même année, le Commentatio delle più notabili et mostruose cose d'Italia, il reprend la stratégie du récit-cadre. Cette œuvre est une description plus ou moins fantaisiste de toutes les choses remarquables que l'on peut trouver dans la péninsule, fait par un navigateur étranger, lequel a donc un point de vue extérieur et qui, ce n'est pas un hasard, est de retour d'un voyage dans l'île d'Utopie. Son navire, poussé par des vents 
contraires, est chargé de carottes : or, dans le vocabulaire italien de la Renaissance, « dire des carottes » signifiait « raconter des bêtises ».

Dans de belles pages, Jean-Claude Margolin (2002: 269-292) relie la tradition utopique à l'union entre les catégories de sagesse et de folie telles qu'elles ont été abordées dans l' Éloge de la Folie d'Érasme qui dénonce la relativité de tout point de vue. Si les solutions utopiques nous paraissent absurdes, c'est seulement parce que notre esprit est incapable de voir au-delà des circonstances qui lui sont imposées, de sorte que beaucoup de revendications sociales qui auraient semblé impraticables à d'autres époques ne le sont pas aujourd'hui. L'impossibilité pour l'homme d'opérer un tel changement de perspective est compensée par la création du monde à l'envers et c'est grâce à cette fiction littéraire qu'il est possible de saisir une réalité plus optimiste, que le monde actuel, divisé par les luttes et la discorde, a effacée. Sans cette réflexion de fond, les utopies ont été facilement considérées soit comme des programmes immédiatement réalisables, soit comme des rêves complètement détachés du contexte social et politique, alors que la réalité réside plutôt entre ces deux positions extrêmes. More situe son île imaginaire dans un monde complètement abstrait non parce qu'il ne prenait pas en compte, ou ignorait, la réalité de son temps mais parce que justement il voulait s'éloigner des excès et de la corruption d'un monde qui est savant grâce à l'esprit pratique des hommes politiques, mais fou parce que la raison et le bon sens n'y trouvent plus leur place. Margolin attire l'attention sur une définition de la ville utopique comme plan rationnel fondé sur des lois naturelles et non pas sur la raison d'État déterminée par les circonstances historico-politiques. Il faudrait donc considérer l'utopie comme extérieure à l'Histoire, non par incapacité à secouer les consciences ou à déclencher des révolutions, mais en raison du fait qu'elle en refuse les normes amorales et se donne comme aspiration un monde meilleur qui ne se rabaisse pas aux lois de la ratio status, mais suit la raison et le bon sens, lesquels la rendent exportable à l'échelle universelle, comme le rêvait Campanella.

\section{BIBLIOGRAPHIE}

BERTANA Emilio (1892), « Un socialista del Cinquecento. Appunti sulla vita e sugli scritti d'Antonfrancesco Doni », Giornale Ligustico di archeologia, storia e letteratura, XIX, 336-372.

BotTAI Giuseppe et DE LuCA Giuseppe (1989), Carteggio, 1940-1957, Renzo de Felice et Renato Moro (éds), Rome : Ed. di storia e letteratura.

CALENDA Di TAVANI Andrea (1895), Fra' Tommaso Campanella e la sua dottrina sociale e politica di fronte al socialismo moderno, Nocera Inferiore : A. Angora.

CANTIMORI Delio (1943), Utopisti e riformatori italiani: 1794-1847, Florence : Sansoni.

CROCE Benedetto (1901), Matérialisme historique et économie marxiste (Alfred Bonnet, trad.), Paris : V. Giard \& E. Bière. (Ouvrage original publié en 1900 sous le titre Materialismo storico e economia marxista, Milan, Palerme : R. Sandron.)

CROCE Benedetto (1948), Come il Marx fece passare il comunismo dall'utopia alla scienza, Bari : Laterza. 
CuRcio Carlo (éd.) (1941), Utopisti e riformatori sociali del Cinquecento, Bologne : Zanichelli.

Doni Anton Francesco (1994), I Mondi e gli Inferni [1552], Patrizia Pellizzari (éd.), Turin : Einaudi.

FIRPo Luigi (1957), Lo stato ideale della Controriforma: Ludovico Agostini, Bari : Laterza.

JAMESON Fredric (2007), Archéologies du futur. I : Le désir nommé utopie (Nicolas Vieillescazes et Fabien Ollier, trad.), Paris : M. Milo. (Ouvrage original publié en 2005 avec le titre Archeologies of the Future: The Desire Called Utopia and Other Science Fictions, New York : Verso.)

LABRIOLA Antonio (1970), Essais sur la conception matérialiste de l'histoire (Alfred Bonnet, trad.) [1902], Paris, Londres, New York : Gordon \& Breach. (Ouvrages originaux publiés en 1895 et 1896 sous le titre In memoria del manifesto dei comunisti et Del materialismo storico, Rome : Loescher.)

LAFARGUe Paul (1895), «Campanella. Étude critique sur sa vie et sur la Cité du Soleil », Le Devenir social, 4-6, 305-320, 465-480, 561-578.

LAVELEYE Émile DE (1883), Le socialisme contemporain, Paris : Germer-Baillière.

MALON Benoît (1880), Histoire du socialisme depuis les temps les plus reculés jusqu'à nos jours. Vol. I : Histoire du socialisme, depuis ses origines probables jusqu'à la fin de la Révolution française, Lyon : H. Albert.

MALON Benoît (1892a), Le socialisme intégral (t. I), Paris : F. Alcan.

MALON Benoît (1892b), Précis historique, théorique et pratique de socialisme, Paris : F. Alcan.

MARGOLIN Jean-Claude (2002), « Erasmo da Rotterdam e la respublica litterarum umanistica della prima metà del XVI secolo. Sapienza, "follia" e utopia nell'Europa delle grandi guerre e dei conflitti religiosi », «Le forme della conservazione politica: ragion di Stato e utopia », C. Vasoli \& P. C. Pissavino (éds), Le filosofie del Rinascimento, Milan : Mondadori, 269-292.

MELCHIORRE Virgilio (1970), La coscienza utopica, Milan : Vita e pensiero.

PISSAvino Paolo Costantino (2002), « Le forme della conservazione politica: ragion di Stato e utopia », C. Vasoli \& P. C. Pissavino (éds), Le filosofie del Rinascimento, Milan : Mondadori, 552-606. QUARTA Cosimo (2015), Homo utopicus. La dimensione storico-antropologica dell'utopia, Bari : Dedalo. RivoletTi Christian (2003), Le metamorfosi dell'utopia. Anton Francesco Doni e l'immaginario utopico di metà Cinquecento, Lucca : M. Pacini Fazzi.

Villegardelle François (1840), « Notice sur Campanella », Tommaso Campanella, La Cité du Soleil, ou Idée d'une République philosophique (F. Villegardelle, trad.), Paris : Alphonse Levavasseur.

Widmar Bruno (éd.) (1964), Scrittori politici del ‘500 e del ‘600, Milan : Rizzoli.

\section{NOTES}

1. À propos du rapport entre utopie et politique, voir Jameson (2007).

2. Comme nous le savons, le sens du mot « utopia» peut être considéré comme la traduction

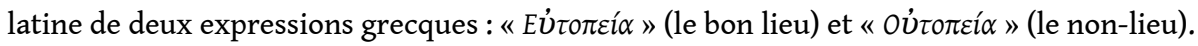

3. Voir par exemple Quarta (2015).

4. Citation de Michel de Montaigne, Les Essais, III, 2 et Malon (1829b : 3).

5. Nous traduisons.

6. Publié initialement en 1896 dans Atti dell'Accademia pontaniana di Napoli, vol. XXVI, maintenant dans Croce (1921 : 1-20) sous le titre de Sulla forma scientifica del materialismo storico. 
7. «Il comunismo di Tommaso Campanella », publié initialement en 1895 dans Archivio storico per le provincie napoletane, XX, IV, maintenant dans Croce (1921: 177-216).

8. Nous traduisons.

9. Parmi les volumes sur la Renaissance publiés dans la collection «Scrittori politici italiani » de l'INCF, nous pouvons mentionner : Giovanni Francesco Lottini, Avvedimenti civili ; Orazioni politiche del '500, avec des textes d'Alamanni, Bandini, Bembo, Cavalcanti, Della Casa, Guidiccioni, Lollio, Lorenzino de' Medici, Nardi, Tolmei ; Coluccio Salutati, Il trattato De Tyranno e lettere scelte ; Leon Battista Alberti, Momus o del principe ; Paolo Pauta, Discorsi politici.

10. Nous traduisons.

11. Nous retrouvons les auteurs présents dans la collection de l'INCF : Doni, Patrizi, Foglietta, Agostini ( $\left.\mathrm{n}^{\circ} 19\right)$, Lottini ( $\left.\mathrm{n}^{\circ} 22\right)$ avec l'ajout de Della perfezione della vita politica de Paolo Paruta, de Della Ragion di Stato de Giovanni Botero, de La Cité du Soleil de Campanella, de La libertà ecclesiastica de Paolo Sarpi, de Il Belluzzi ou La città felice de Lodovico Zuccolo.

12. Des voyants ayant prévu, grâce à des observations astrologiques, l'arrivée d'une forte pluie qui aurait rendu folle toute la population, ils décidèrent de se réfugier dans une casemate avec l'idée de prendre le contrôle du territoire, une fois qu'ils seraient restés les seules personnes savantes. Toutefois, quand ils sortirent de leur refuge, leur comportement est considéré comme insensé par les fous et ils sont obligés de se conformer à la folie (Doni, 1994 : 158-161).

\section{RÉSUMÉS}

La pensée utopique, telle qu'elle a été définie par certains philosophes et historiens, serait le moteur de tous les changements sociaux qui ont fini par déterminer notre Histoire. Dans ce sens, les utopies de la Renaissance ont été interprétées par des socialistes comme le germe des mouvements politiques modernes, alors que Benedetto Croce, dans sa vision de l'histoire comme action, attachera peu d'importance à l'utopie qu'il considère plutôt comme l'expression d'une tendance à la stase et à la conservation. Malgré cette position crocienne, partagée par Karl Mannheim et d'autres intellectuels, nous verrons que les utopies littéraires ont continué à avoir une place importante dans l'histoire de la pensée politique.

La présente contribution vise donc à retracer les étapes de la réception hétérogène des textes utopiques italiens, en particulier des Mondes de Doni et de La Cité du Soleil de Campanella, en se demandant si la forme ambiguë, entre fiction et réalité, du roman utopique moderne conçu par More a joué un rôle dans cette vision dissonante des ouvrages.

Utopian thought has been defined by some philosophers and historians as the driving force behind all the social changes that have determined the course of history. While, in this perspective, Renaissance utopias have been interpreted by some socialists thinkers as the seed of modern political movements, Benedetto Croce, in accordance with his view of history as action, attaches less importance to utopia, which he considers as a charged with a tendency to stasis and conservation. Despite the significance of the Crocian conception of utopia, which was also shared by Karl Mannheim and others intellectuals, it can be shown that literary utopias have continued to play an important role in the history of political thought.

This contribution intends to retrace the stages of the heterogeneous reception of Italian utopian texts, especially Doni's Mondi and Campanella's La Città del Sole, and wonders whether the 
ambiguous form of the utopian modern novel invented by More, between fiction and reality, played a role in this dissonant interpretation of such works.

INDEX

Mots-clés : utopie, Renaissance italienne, matérialisme historique, Campanella Tommaso, Doni Anton Francesco

Keywords : utopia, Italian Renaissance, historical materialism, Campanella Tommaso, Doni Anton Francesco

\section{AUTEUR}

FEDERICA GRECO

Université Grenoble-Alpes 